\title{
Saturated fat-rich diet enhances selective uptake of LDL cholesteryl esters in the arterial wall
}

\author{
Toru Seo, ${ }^{1}$ Kemin Qi, ${ }^{1}$ Chuchun Chang, ${ }^{1}$ Ying Liu, ${ }^{1}$ Tilla S. Worgall, ${ }^{2}$ \\ Rajasekhar Ramakrishnan, ${ }^{1}$ and Richard J. Deckelbaum ${ }^{1}$ \\ 1Department of Pediatrics and 2Department of Pathology, Institute of Human Nutrition, College of Physicians and Surgeons, \\ Columbia University, New York, New York, USA.
}

\begin{abstract}
Plasma LDL levels and atherosclerosis both increase on a saturated fat-rich (SAT) diet. LDL cholesterol delivery to tissue may occur via uptake of the LDL particles or via selective uptake (SU), wherein cholesteryl ester (CE) enters cells without concomitant whole-particle uptake. It is not known how dietary fats might directly affect arterial LDL-CE uptake and whether SU is involved. Thus, mice that are relatively atherosclerosis resistant $(\mathrm{C} 57 \mathrm{BL} / 6)$ or susceptible to atherosclerosis $\left(\mathrm{apoE}^{-/-}\right)$were fed a chow or SAT diet and injected with double radiolabeled or fluorescent-labeled human LDL to independently trace LDL-CE core and whole-particle uptake, respectively. Our results show that a SAT diet increased contributions of SU to total arterial LDL-CE delivery in $\mathrm{C} 57 \mathrm{BL} / 6$ and apo $\mathrm{E}^{-/-}$mice. The SAT diet increased plasma fatty acid and cholesterol levels; cholesterol, but not fatty acid, levels correlated with SU, as did the degree of atherosclerosis. Increased SU did not correlate with arterial scavenger receptor class B type I levels but paralleled increased lipoprotein lipase (LPL) levels and LPL distribution in the arterial wall. These studies suggest that arterial LDL-CE delivery via SU can be an important mechanism in vivo and that dietary influences on arterial LPL levels and atherogenesis modulate arterial LDL-CE delivery, cholesterol deposition, and SU.
\end{abstract}

\section{Introduction}

Although the LDL receptor (LDLR) is a major pathway for systemic LDL clearance and for cholesterol delivery to many cell types (1), cholesterol accumulation in the arterial wall with limited LDLR expression indicates that cholesterol delivery is mediated by mechanisms independent of the LDLR. Pathologic arterial lipid accumulation, an important contributor to atherogenesis, is linked to a number of pathways involving CD36 or other scavenger receptors that bind to normal or modified $\operatorname{LDL}(2,3)$ to promote the progression of atherosclerosis $(4,5)$. Moreover, cell surface proteoglycans that are ubiquitously expressed in cells have been shown to mediate LDL uptake via low-affinity but high-capacity processes (6), which leads to substantial cholesterol accumulation in cells. These processes have generally been studied by evaluating apoB uptake, an indicator for LDL cholesterol uptake.

LDL cholesteryl esters (CEs) can be independently delivered to cells without concomitant uptake of the whole LDL particle, a process named "selective uptake" (SU) (7-9). SU leads to accumulation of cholesterol in cells and tissues that exceeds cholesterol delivery accounted for by whole-particle uptake. SU from HDL via scavenger receptor type B-I (SR-BI) has been well characterized (10) and is linked to steroidogenesis (11) and reverse cholesterol transport (12). Unlike those of HDL SU, the pathways and physiological significance of LDL SU are less defined. However, studies by Green and Pittman (7) indicated that LDL SU occurs in lipo-

Nonstandard abbreviations used: CE, cholesteryl ester; $d$, density fraction; LPL, lipoprotein lipase; SAT, saturated fat-rich; SR-BI, scavenger receptor class B type I; SU, selective uptake; TC, tyramine cellobiose; TG, triglyceride.

Conflict of interest: The authors have declared that no conflict of interest exists.

Citation for this article: J. Clin. Invest. 115:2214-2222 (2005).

doi:10.1172/JCI24327. protein lipase-rich (LPL-rich) tissues such as muscle, heart, and adipose tissues. In keeping with these observations, LDL-SU in muscle is significantly increased in mice overexpressing human LPL in muscle (9).

We previously demonstrated that non-SR-BI-mediated SU in cultured macrophages was markedly enhanced by FFAs (13). Thus, it is possible that alterations in plasma FFA levels and/or composition, induced by diet or by disease (e.g., diabetes), lead to increased cholesterol delivery via SU to the arterial wall. FFAs and cholesterol often interact and influence systemic lipid homeostasis and LDL cholesterol metabolic pathways (14). Specific dietary FFAs may directly modulate cholesterol delivery to extrahepatic tissues, such as the arterial wall, influencing the progression of atherosclerosis.

In the studies described herein, we compared the role of SU in arterial LDL-CE delivery in relatively atherosclerosis-resistant C57BL/ 6 and atherosclerosis-susceptible apoE $/-$ fed a chow or a SAT diet. We show that SU contributes substantially to arterial LDL-CE delivery in both mouse groups, especially with a SAT diet. We found that changes in plasma cholesterol levels were a key factor that influences arterial LDL-CE delivery via SU. Moreover, SU was associated with increases in plasma cholesterol concentrations, arterial LPL levels, and extent of atherosclerosis, which suggests that these factors can interact to increase arterial cholesterol deposition via SU.

\section{Results}

Effect of diet on plasma lipids and arterial neutral lipid staining. We followed the effects of dietary saturated fats on plasma lipid profiles with a chow or SAT feeding initiated 4 weeks after birth. Plasma cholesterol, triglyceride (TG), and FFA levels were determined every week for the first 4 weeks after feeding and every other week thereafter. Changes in plasma lipid profiles occurred in the first 4 


\section{Table 1}

Plasma lipid profiles of C57BL/6 and apo $\mathrm{E}^{-/-}$mice fed a chow or SAT diet

\begin{tabular}{lcccccc} 
& \multicolumn{3}{c}{ C57BL/6 } & & \multicolumn{2}{c}{ apoE ${ }^{-/-}$} \\
Feeding period & TC $(\mathbf{m g} / \mathbf{d l})$ & TG $(\mathbf{m g} / \mathbf{d l})$ & FFA $(\mathbf{m M})$ & TC $(\mathbf{m g} / \mathbf{d l})$ & TG $(\mathbf{m g} / \mathbf{d l})$ & FFA $(\mathbf{m M})$ \\
4-wk chow & $48.10 \pm 13.77$ & $38.45 \pm 18.27$ & $0.29 \pm 0.05$ & $239.85 \pm 35.22$ & $48.99 \pm 22.16$ & $0.23 \pm 0.93$ \\
4-wk SAT & $107.47 \pm 25.10^{\mathrm{A}}$ & $80.01 \pm 14.88$ & $0.55 \pm 0.10$ & $402.71 \pm 55.01^{\mathrm{A}}$ & $76.45 \pm 13.19$ & $0.44 \pm 0.15^{\mathrm{A}}$ \\
12-wk chow & $52.04 \pm 10.92$ & $43.11 \pm 12.20$ & $0.24 \pm 0.09$ & $259.50 \pm 56.74$ & $58.35 \pm 18.63$ & $0.38 \pm 0.26$ \\
12-wk SAT & $120.58 \pm 19.45^{\mathrm{A}}$ & $77.47 \pm 10.15$ & $0.47 \pm 0.12$ & $663.57 \pm 154.33^{\mathrm{A}}$ & $83.55 \pm 21.01$ & $0.52 \pm 0.22^{\mathrm{A}}$ \\
\hline
\end{tabular}

mined for total cholesterol (TC), TG, and FFA levels as described in Methods. ASignificant differences between chow- and SAT-fed mice.

weeks after feeding regimens were initiated in $\mathrm{C} 57 \mathrm{BL} / 6$ and $\mathrm{apoE}^{-/-}$ mice. The SAT feeding for 4 weeks increased cholesterol and TG levels in C57BL/6 mice 2.3- and 2-fold, compared with chow feeding (Table 1). In these mice, cholesterol and TG levels remained elevated and changed little after 12 weeks on the SAT diet. However, cholesterol levels in apoE $\mathrm{E}^{-/-}$mice more than doubled with the SAT diet after 4 and 12 weeks, with mice fed the SAT diet for 12 weeks having higher cholesterol levels than those fed for 4 weeks. There were mild to moderate increases in plasma FFA levels in all groups within the first 4 weeks, but FFA levels increased much more (>2-fold) in the mice fed the SAT diet (Figure 1). On the SAT diet, FFA levels remained elevated during the entire 12-week feeding period (Table 1).

At the end of the feeding periods, aortas were isolated, stained for lipid accumulation with oil red $\mathrm{O}$, and examined under an inverted light microscope. As characterized previously by other laboratories (15), a $\mathrm{poE}^{-/-}$mice developed obvious atherosclerotic lesions, with the extent and severity increasing with age and duration of SAT feeding. Aortas from apoE $\mathrm{E}^{-/-}$mice on a chow or SAT diet for 4 weeks showed little or no staining, while apoE-/- mice fed the SAT diet for 12 weeks had disrupted aortas with advanced atherosclerotic plaques with necrotic cores. In contrast, no visible lesions were observed in C57BL/ 6 mice independent of diet (data not shown).

Dietary effects on $L D L-C E$ and $a p o B$ clearance in $C 57 B L / 6$ and apo $E^{-1-}$ mice. To determine possible SAT diet effects on the blood clearance of injected LDL in C57BL/ 6 and apoE $\mathrm{E}^{-/-}$mice, ${ }^{3} \mathrm{H}$ and ${ }^{125} \mathrm{I}$ radioactivity (markers for LDL-CE and apoB, respectively) were independently measured at 7 different time points over 24 hours. In C57BL/6 mice, there were no significant differences in blood LDL-CE or particle clearance in mice fed a chow or SAT diet (Figure 2A), and fractional catabolic rates (FCRs) were very similar (Figure 2A, inset). In contrast, apoE $\mathrm{E}^{-/}$mice had apparent slower blood LDL clearances as compared with $\mathrm{C} 57 \mathrm{BL} / 6$ mice, and this rate was further reduced in mice fed the SAT diet (Figure 2B), a result most likely due to the increased level of endogenous apoB-containing lipoproteins competing with injected LDL for blood clearance. There were no significant differences in blood clearance between the ${ }^{3} \mathrm{H}$ and ${ }^{125} \mathrm{I}$ radiolabels at each time point in all mouse groups. These studies suggest that increased cholesterol levels in apoE ${ }^{-/-}$mice reduced LDL clearance without affecting SU and that SU from LDL does not contribute significantly to clearance of LDL-CE from the plasma compartment.

Contribution of SU to cholesterol delivery to tissues and CE accumulation in the arterial wall. To determine whether SU contributed to CE delivery to various tissues, we sacrificed mice fed chow and SAT diets for 4 and 12 weeks 24 hours after LDL injection and determined radioactivity in organs and tissues. Of total injected LDL,
$45-72 \%$ was recovered in the liver. Consistent with results of previous studies (13), SU contributed to LDL-CE delivery to some tissues (i.e., liver, spleen, muscle). In the liver, SU contributed $16.2 \%$ and $14.2 \%$ to total LDL-CE uptake in chow- and SAT-fed mice, respectively. SU contributed $23.7 \%$ and $22.1 \%$ in spleen of chowversus SAT-fed mice, while SU in muscle accounted for $7.9 \%$ and $11.6 \%$ of total LDL-CE uptake. Despite the apparent SU in these tissues, SU was not significantly different between chow and SAT feeding or between mouse types.

In contrast, in C57BL/ 6 mice fed an SAT versus a chow diet for 4 weeks (Figure 3 ), the contribution of SU to total CE delivery in the arterial wall was doubled, accounting for almost $15 \%$ of total arterial LDL-CE uptake. Moreover, after 12-week feeding periods, apoE $/ /-$ mice had higher contribution of SU even on a chow diet (C56BL/6, 7.5\% vs. apoE $\mathrm{E}^{-/}, 20.2 \% ; P<0.02$ ). The contribution of SU increased substantially when mice were on the SAT diet for longer time periods. In apoE $\mathrm{E}^{-/-}$mice fed the SAT diet for 12 weeks, SU contributed up to almost half of total arterial LDL-CE uptake. This suggests that longer SAT feeding leads to increased arterial SU in both $\mathrm{C} 57 \mathrm{BL} / 6$ and apoE $\mathrm{E}^{-/-}$mice. Since atherosclerotic changes were much less or absent in apoE $\mathrm{E}^{-/}$mice fed the SAT diet for 4 weeks compared with apoE $\mathrm{E}^{-/-}$mice fed the diet for 12 weeks, the extent and severity of atherosclerosis likely also affects arterial CE delivery via SU. Thus, these studies indicate that SU from LDL can be an important mechanism for arterial CE delivery in vivo. SU is enhanced by SAT feeding and is also linked to the severity of atherosclerosis.

Correlation of plasma FFAs and cholesterol levels with arterial SU. The SAT diet increases plasma FFA and total cholesterol levels. We asked whether changes in plasma FFA and/or cholesterol levels
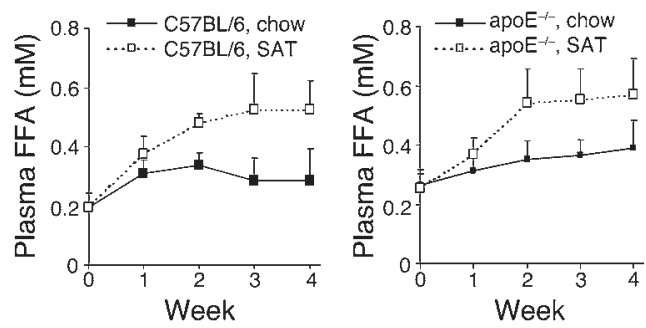

\section{Figure 1}

Effects of diet on plasma FFA levels in C57BL/6 and apoE-/- mice. Blood samples were collected to assess changes in plasma FFA levels as described in Methods. Only FFA levels during first 4 weeks are shown. Each point indicates the mean plasma FFA levels of 20-30 mice from each group \pm SD. 

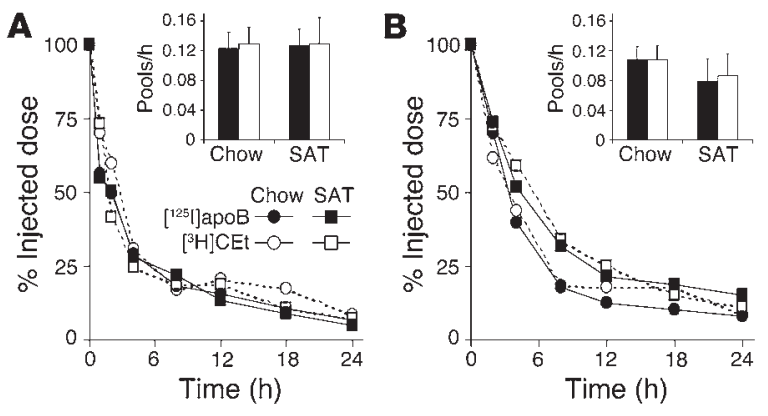

Figure 2

Plasma clearance rate and FCR of LDL-CE core and whole particle of injected LDL in C57BL/6 and apoE--- mice fed a chow or SAT diet. Four-week-old C57BL/6 (A) and apoE ${ }^{-/-}$mice (B) were fed a chow or SAT diet for 12 weeks and then injected with double-labeled LDL as described in Methods. Plasma clearance of ${ }^{3} \mathrm{H}$ and ${ }^{125} \mathrm{I}$ of injected LDL were independently measured to trace clearance of apoB (measured by whole-particle uptake; filled symbols) and clearance of LDLCE (open symbols). Squares and circles indicate mice fed chow or SAT diet, respectively. Results are expressed as percent injected LDL radioactivity remaining in the plasma at each time point over 24 hours. Each data point indicates the mean radioactivity present in blood measured from a minimum of 5 mice. Insets: FCR determined from blood clearance of ${ }^{125}$ (open bars) and ${ }^{3} \mathrm{H}$ (filled bars), using slopes that follow the first-order kinetics.

were associated with increased contribution of SU to total arterial CE delivery. We calculated and plotted the mean values of FFA and cholesterol levels for each set of experiments against those of arterial SU. As shown in Figure 4A, no correlations between plasma FFA levels and SU were demonstrated $\left(r^{2}=0.097 ; P=0.45\right)$. In contrast, Figure 4B shows a close correlation between arterial SU and plasma cholesterol levels $\left(r^{2}=0.815, P=0.002\right)$. Further analyses of plasma cholesterol levels and SU within and among groups (Figure 5) revealed that plasma cholesterol associated closely with changes in SU contribution to arterial LDL-CE uptake in mice fed these diets for 4 weeks (Figure 5A). The effect was not influenced by diet and/or mouse strains, as regression lines at 4 weeks for each group superimposed. Plasma cholesterol levels also influenced SU in mice fed both diets for 12 weeks. However, additional factors that seem to be associated with mouse strains and/or diet augmented changes in SU. The contributions of modifiers in addition to plasma cholesterol levels is suggested by regression lines with very similar slopes but different $y$-intercepts (Figure 5B). For example, the higher $y$-axis intercepts for apoE-/- mice suggest that changes associated with absence of apoE (e.g., atherosclerosis) also contribute to increased SU. Taken together, these studies suggested that plasma cholesterol but not FFA levels were closely related to the contribution of SU to arterial LDL-CE delivery.

Differential LDL uptake and SU in specific areas of the arterial wall. To determine how diets and mouse strains influence apoB and CE deposition in specific areas of aortas, we measured LDL uptake and SU using double-labeled fluorescent LDL in mice that had been fed a chow or SAT diet for 12 weeks (Figure 6). LDL was labeled with BODIPY- $\mathrm{C}_{12}$ and ALEXA to trace LDL-CE and apoB, respectively. Use of fluorescent LDL allowed us to determine which specific layers of the arterial wall (i.e., intima, media, etc.) were associated with increased LDL-CE accumulation. Also, this method enabled us to compare LDL uptake, and SU, in proximal and distal arteries, which was not possible with isotopic methods due to the fact that accumulation of radioactivity in individual aortic segments is limited.

Although the SAT diet in C57BL/6 mice had little effect on apoB-ALEXA deposition compared with chow diet, the uptake of LDL-CE, as traced by BODIPY-C $\mathrm{C}_{12}$, was markedly increased by the SAT diet, as demonstrated by the greener color of the fluorescence in the superimposed images (Figure 6, F vs. C). The brighter and more diffuse green fluorescence throughout the proximal aorta indicates significantly higher LDL-CE infiltration into the medial layer. This parallels the increased arterial CE/apoB radiolabel ratios in SAT-fed mice and is in keeping with the finding that the SAT diet increases SU. In chow-fed apoE $\mathrm{E}^{-/}$mice, there was consistently higher LDL-CE uptake relative to apoB uptake compared with C57BL/6 mice (Figure 6, H vs. B) as well as overall greener color of the fluorescence in the overlays (Figure 6, I vs. C), again in keeping with higher $\mathrm{SU}$ in apoE $\mathrm{E}^{-/-}$mice. In apoE $\mathrm{E}^{-/-}$mice fed the SAT diet for 12 weeks and with atherosclerosis, apoB and LDLCE concentrated and colocalized in the atherosclerotic lesions, as shown by punctuated patterns in the lesion areas (Figure 6, J-L). In these mice, LDL-CE fluorescence was also observed throughout the aorta, with much less apoB colocalization (Figure 6, K and L). In all mice tested, these differences were only observed in the proximal aortas. LDL-CE and apoB uptake were similar, as were relative $\mathrm{CE} / \mathrm{apoB}$ ratios regardless of diet in distal aortas. These results are consistent with the higher LDL flux and the increased atherosclerosis susceptibility in proximal compared with distal aortas.

Effects of dietary fats on arterial LPL and SR-BI expression. We and others have previously demonstrated that LPL stimulates SU from LDL in in vitro and in vivo studies $(9,16)$. Since LPL is enriched in certain tissues by SAT feeding $(17,18)$, we explored whether increased arterial LDL delivery and SU in mice fed the SAT diet corresponded with arterial LPL expression. We also determined SR-BI levels in the arterial wall, since SR-BI has been shown to mediate LDL-SU in vitro under certain conditions. As shown in Figure 7, C57BL/6 mice and apoE $\mathrm{E}^{-/-}$mice fed the SAT diet for 12 weeks had significant increases in LPL levels compared with chow-fed mice. The ratios of LPL density in SAT-fed relative to chow-fed mice were $2.54 \pm 0.21$ and $1.50 \pm 0.37$ for $\mathrm{C} 57 \mathrm{BL} / 6$ and apoE ${ }^{-/-}$mice, respec-

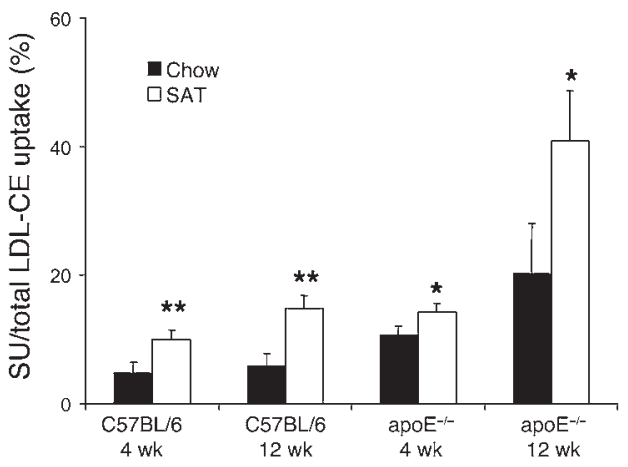

Figure 3

Contribution of SU to total arterial LDL-CE delivery. Isolated arteries from C57BL/6 and apoE ${ }^{-/}$mice fed a chow (black bars) or SAT (white bars) diet were measured for ${ }^{3} \mathrm{H}$ and ${ }^{125} \mathrm{I}$ radioactivity as described in Methods. Percent contribution of SU to total CE delivery was determined from differences in LDL-CE uptake calculated from ${ }^{3} \mathrm{H}$ and ${ }^{125}$ radioactivity (SU) compared with total LDL-CE uptake. The results are expressed as the mean $\pm \mathrm{SD}$, where sample size in each group was at least 15 mice. ${ }^{*} P<0.05$ and ${ }^{* *} P<0.02$, SAT vs. chow. 


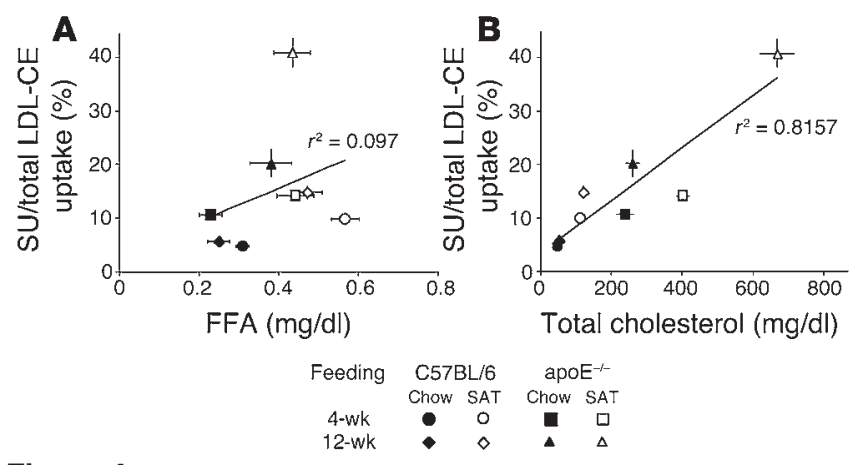

Figure 4

Relationship between plasma FFA and cholesterol levels and changes in arterial SU. Averaged plasma FFA (A) and cholesterol levels (B) from $\mathrm{C} 57 \mathrm{BL} / 6$ and apo: ${ }^{-/}$mice in each diet group were plotted against the contribution of percent SU to total arterial LDL-CE delivery. $x$ - and $y$-axis error bars show SEM of the mean in each group ( $n=13-30$ for each point).

tively $(n=3-5 ; P<0.05)$. Increased LPL was associated with higher proximal aorta LPL activity in SAT-fed mice, as determined by the hydrolysis of Intralipid emulsions (C57BL/6: chow, $22.5 \pm 3.5$ vs. SAT, $165.0 \pm 12.2$ units FFA released/mg artery protein; apoE-/-: chow, $14.3 \pm 5.2$ vs. SAT, $91.4 \pm 24.3$ units FFA released $/ \mathrm{mg}$ artery protein). Of interest, differences in LPL activity in mice fed a SAT diet were highly significant in proximal aortas $(P<0.01)$ but not in distal aortas in both mouse groups (data not shown).

Although there were substantial amounts of SR-BI expressed in the arterial wall in both $\mathrm{C} 57 \mathrm{BL} / 6$ and $\mathrm{apoE}^{-/-}$mice, there were no significant effects on SR-BI expression induced by different diets (SAT/chow ratios, $1.25 \pm 0.54$ and $0.77 \pm 0.74$ for $\mathrm{C} 57 \mathrm{BL} / 6$ and apoE $\mathrm{E}^{-/}$mice, respectively; $n=3-5$ ), which suggests that SR-BI in the arterial wall is not an important contributor to the increased SU associated with the SAT diet.

To further examine the relationships between arterial LPL expression and increased LDL-CE uptake in C57BL/6 mice and apoE-/mice fed the SAT diet, we performed immunohistochemical staining of arterial LPL (Figure 8) to determine whether its distribution patterns related to those of fluorescent LDL-apoB and LDL$\mathrm{CE}$ (Figure 6). In C57BL/6 mice, there were increases in arterial LPL on the SAT diet, consistent with results from the Western blot analyses (Figure 8A). In younger C57BL/6 mice fed a chow or SAT diet for 4 weeks, LPL was distributed mainly in intimal layers $(197.0 \pm 28.2$ vs. $286.1 \pm 40.1$ mean density per area \pm SEM, respectively; $n=4 ; P<0.03)$. Mice fed for 12 weeks had higher LPL deposition throughout medial layers in the arterial wall, especially on the SAT $\operatorname{diet}(237.9 \pm 39.2$ vs. $361.9 \pm 62.2$ mean density per area \pm SEM, respectively; $n=6 ; P<0.02$ ). Although there was less arterial LPL immunoreactivity in apoE $\mathrm{E}^{-/-}$mice than in $\mathrm{C} 57 \mathrm{BL} / 6$ mice at the end of the 4-week feeding period (Figure 8B), the SAT diet still more than doubled LPL levels in these mice (chow, $43.0 \pm 28.2$ vs. SAT, $113.1 \pm 22.1$ mean density per area \pm SEM; $n=5 ; P<0.05$ ). The increased LPL expression paralleled a 2.2 -fold increase in LPL activity. (In apoE $/ /$ - mice, differences in arterial LPL expression and activity levels could not be documented when mice were maintained for 12 weeks on a chow or SAT diet due to extensive disruption of the arterial wall accompanying atherosclerosis and the presence of large amounts of noncellular debris within the artery.) Collectively, these studies indicated that dietary fats can influence arterial LPL levels and that this varied with the length of feeding periods and mouse strains. Since arterial LPL levels and distribution were consistent with the amount and the localization of the LDL-CE tracer BODIPY-C ${ }_{12}$ (compare Figure 8 and Figure 6) in C57BL/6 mice and in younger apoE-/- mice, we propose that arterial LPL contributes to increased arterial LDL uptake and SU, especially in mice fed a SAT diet.

\section{Discussion}

Although SU from LDL occurs both in vivo and in vitro and can play a role in cholesterol delivery to tissues that have little or no LDL receptors, physiological and pathophysiological roles for LDL SU have not been fully delineated. In the studies described herein, we examined how a diet rich in saturated fat modulates SU from LDL and influences LDL clearance and arterial CE deposition using atherosclerosis-resistant C57BL/6 and -susceptible apoE $\mathrm{E}^{-/-}$mice. We assessed the effects of saturated fat on arterial SU and its possible links to plasma FFA and cholesterol levels. We also asked how the extent of atherosclerosis contributes to changes in arterial LDL uptake and SU. In the radioisotopic studies, SAT diet increased the contribution of SU to arterial CE delivery, and this increased with longer SAT diet consumption in C57BL/6 mice. In apoE $\mathrm{E}^{-/-}$mice, the contribution of SU was higher even on a chow diet, and the SAT diet increased SU from LDL to account for 40-50\% of arterial CE delivery. Similar findings were obtained in the studies in which we utilized double fluorescent-labeled LDL, and we confirmed that the SAT diet promotes much higher LDLCE deposition than could be accounted for by whole LDL particle uptake. Moreover, higher LDL-CE uptake was associated with more deposition of LDL-CE, which reached into the medial layer in proximal, but not distal, aorta.

In contrast, we found similar clearances for LDL-CE and LDLapoB from the circulation. These findings are different than those of Webb et al. (19), who showed that the plasma clearance of
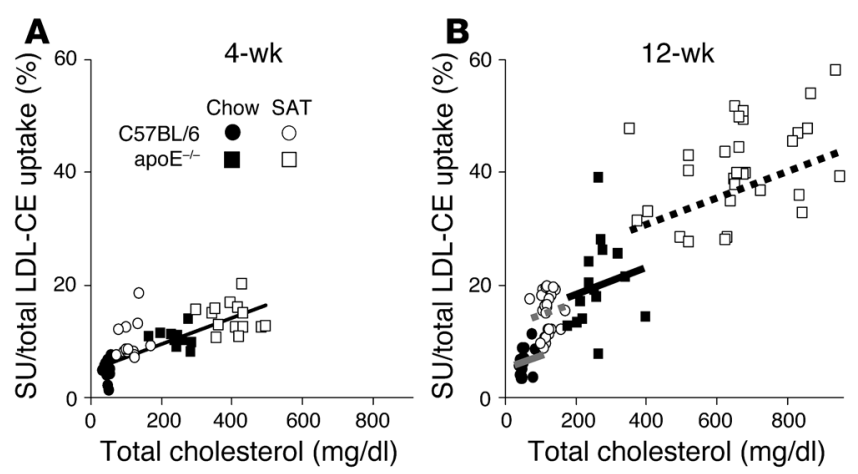

\section{Figure 5}

Interactions of plasma cholesterol levels, diet, and feeding periods on arterial SU. Data for C57BL/6 (circles) and apoE ${ }^{-/}$mice (squares) were plotted individually to demonstrate the effects of interrelationships of plasma cholesterol levels, diet, and feeding period on percent SU relative to total arterial LDL-CE uptake. Data for mice fed a chow or SAT diet for 4 weeks $(\mathbf{A})$ and 12 weeks $(B)$ are indicated by filled and open symbols, respectively. Each regression line was calculated using data points within a group. The solid gray and black lines in B represent the regression lines for chow-fed $\mathrm{C} 57 \mathrm{BL} / 6$ and $\mathrm{apoE}^{-/-}$mice, respectively; the dotted lines indicate those mice fed the SAT diet. Note that regression lines in each group at the 4-week feeding period were identical and thus are represented by a single solid black line in $\mathbf{A}$. 

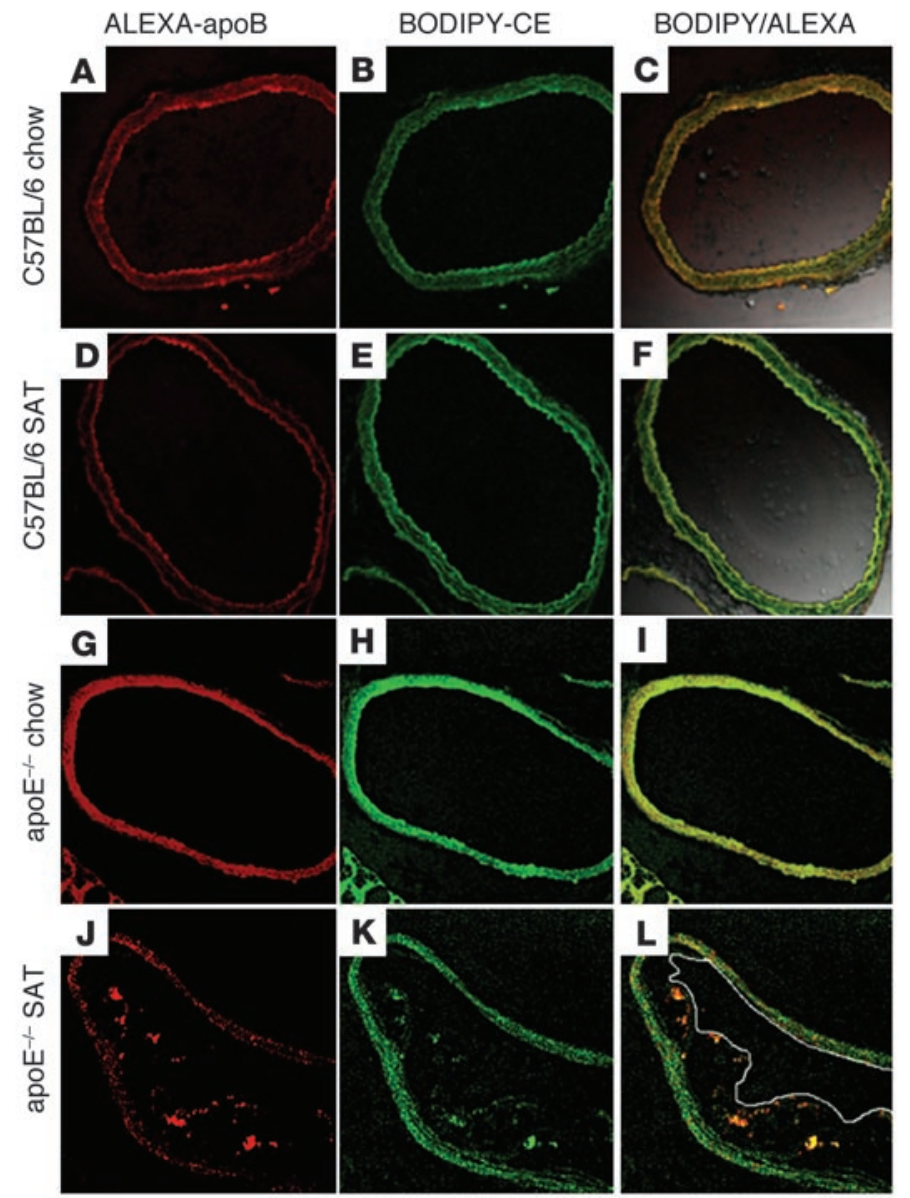

$\left[1 \alpha, 2 \alpha(\mathrm{n})-{ }^{3} \mathrm{H}\right]$ cholesteryl oleoyl ether $\left(\left[{ }^{3} \mathrm{H}\right] \mathrm{CEt}\right)$ (a marker for LDL-CE) was $24 \%$ higher than that of ${ }^{125}$ I (a marker for LDLapoB). These disparate findings were likely due to differences between human LDL used in our studies and mouse LDL used in the studies by Webb et al. In mouse LDL, there is significantly higher apoE content (20) than in human LDL, in which apoE is totally or nearly absent, especially in the density fraction used herein $(\mathrm{d}=1.025-1.055 \mathrm{~g} / \mathrm{ml})$. Since the presence of apoE on LDL increases SU in cultured cells (21), apoE on mouse LDL could lead to increased SU in vivo as well. Indeed, apoE appears to play a major role in mouse LDL-SU pathways, including those mediated by SR-BI. Webb et al. (20) clearly demonstrated that apoB-containing VLDL and LDL from apoE $/ /-$ mice did not undergo SR-BI-mediated SU. These studies are consistent with our previous report that SU from human LDL is mediated by non-SR-BI pathways (9) and now with our current findings that changes in arterial SU do not correlate with levels of SR-BI in the arterial wall. Importantly, changes in dietary feedings and mouse strains alter LDL composition, which, in turn, can influence arterial LDL uptake and/or SU. Thus, by using normal human LDL with relatively constant size and lipid composition, our studies have reduced the potential variability associated with studies using mouse LDL.

Of interest, LDL-CE clearance from the circulation was not influenced by SU from other organs such as liver. Although we found no evidence of LDL loss or efflux from the arterial wall, efflux or "leak" of ${ }^{125}$ I[tyramine cellobiose] ( ${ }^{125}$ I[TC]) labeled apoB fractions

\section{Figure 6}

Effects of diet on fluorescent LDL-CE and apoB uptake in aortas of C57BL/6 $(\mathbf{A}-\mathbf{F})$ and apoE ${ }^{-/-}$mice $(\mathbf{G}-\mathbf{L})$ fed a chow or SAT diet. Eight hours after a bolus injection of double-fluorescent LDL, C57BL/6 mice and apoE ${ }^{-/-}$mice fed for 12 weeks were sacrificed, and fluorescence in the arterial wall was determined as described in Methods. Fluorescent-labeled LDL $(200 \mu \mathrm{g} / \mathrm{ml})$ was labeled with ALEXA (left panels: A, D, G, and J) and BODIPY-C ${ }_{12}$ (middle panels: $\mathbf{B}, \mathbf{E}, \mathbf{H}$, and $\mathbf{K}$ ) to simultaneously trace whole and particle LDL-CE core, respectively. Overlay light microscope images (right panels: $\mathbf{C}, \mathbf{F}, \mathbf{I}$, and $\mathbf{L}$ ) were used to assess colocalization of ALEXA and BODIPY- $\mathrm{C}_{12}$. Each image was captured at the same fixed times for comparison. Experiments were performed on at least 5 different mice from each group, and representative images are shown at $\times 40$ magnification.

of LDL from certain organs such as liver and spleen has been described (22). This would lead to an overestimation on SU in these organs. We have previously demonstrated in in vitro studies that LDL SU has slower kinetics than whole-particle LDL uptake (SU is significant only after 8 hours of incubation of LDL with cultured cells) (13). Since a majority of injected LDL in the circulation was cleared in the first 8 hours after injection and the FCR is strongly influenced at time points under 8 hours, differences in kinetics of LDL-CE clearance from wholeparticles versus SU could also, in part, contribute to the apparent lack of an effect of organ SU on plasma LDL-CE clearance.

Changes in plasma lipid levels associated with SAT feeding included elevated plasma FFA and cholesterol levels in C57BL/6 and apoE - $^{-/}$mice. Correlation analyses of plasma FFA levels with arterial SU showed no significant differences among different experimental groups. In contrast, plasma cholesterol levels highly correlated with the contribution of SU to arterial LDL-CE delivery. Relationships between plasma cholesterol levels and degrees of SU were present under all conditions. However, mice at the 12 -week feeding period, particularly apoE ${ }^{-/-}$mice, showed marked increases in SU that could not be accounted for by cholesterol levels alone. Our statistical models suggest the presence of additional factors (e.g., atherosclerosis itself or processes associated with atherosclerosis) that also promote SU. These factors can be influenced and modified by mouse strains, feeding periods, and diet.

The effects of plasma cholesterol levels on LDL-CE SU are likely mediated by complex interactions at cellular and molecular levels.

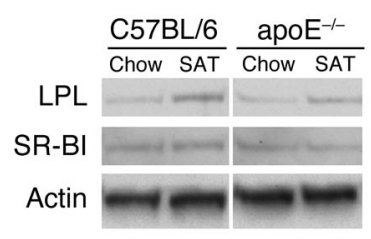

Figure 7

Effects of different diets on LPL and SR-BI in the arterial wall in $\mathrm{C} 57 \mathrm{BL} / 6$ and apoE $\mathrm{E}^{-/-}$mice. Arteries extending from aorta to tracheal artery from C57BL/6 and apo $\mathrm{E}^{-/-}$mice fed a chow or SAT for 12 weeks $(n=3-5)$ were pooled and homogenized for Western blot analyses for LPL and SR-BI. At least 3 Western blots were performed, and they showed very similar results. Changes in arterial LPL or SR-BI expression induced by SAT relative to chow diet were calculated and expressed as relative density change \pm SEM. 
A

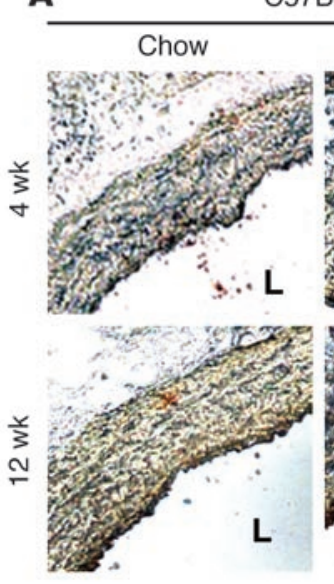

C57BL/6

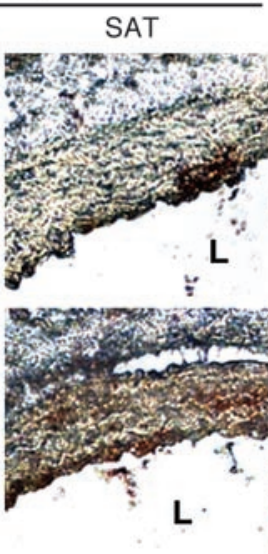

B

Chow

apoE $^{-/-}$

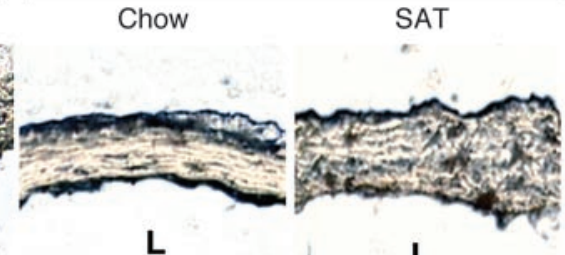

L

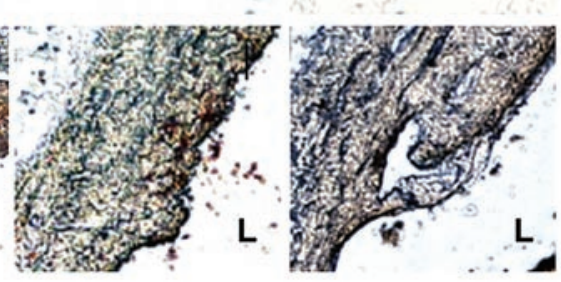

Figure 8

In situ immunohistochemistry of arterial LPL in C57BL/6 and apoE ${ }^{-/-}$mice fed a chow or SAT diet. Isolated arteries from C57BL/6 (A) and apoE ${ }^{-/-}$mice (B) fed a chow or SAT diet for 12 weeks were stained for LPL as described in Methods. Each group consisted of 4-5 mice, and from each artery sample, 10-20 sections were prepared and compared for consistency. Rrepresentative images are shown. Images shown are $\times 200$ magnification, and reddish-brown staining indicates the presence of LPL. The lumen is indicated by $L$.

Possible mechanisms include alteration of cell membrane composition and cell populations in the arterial wall. Tabas (23) demonstrated that free cholesterol accumulation in macrophages causes apoptosis and promotes atherogenesis, conditions that may exacerbate SU and also lead to an increased number of arterial macrophages. Increased cholesterol levels can also directly influence cell membrane properties that are critical for modulating SU in cultured macrophages (13). Although these studies and our current in vitro studies cannot be compared in a straightforward manner, we hypothesize that changes in cholesterol levels can alter cell cholesterol distributions that influence SU. This can occur particularly when metabolically active cells such as macrophages are present in the arterial wall, as is typical in atherosclerotic lesions.

The SAT diet markedly increased levels of arterial LPL, a molecule that promotes LDL delivery and retention ex vivo (24) and mediates CE delivery via SU $(9,16)$. Arterial LPL levels also increased with age, as did SU, and LPL was present throughout intima-medial layers of the arterial wall in older SAT-fed mice. Thus, we speculate that changes in LPL levels within the arterial wall contribute to SU. Feeding rabbits with a high-fat diet has been shown to increase LPL levels in adipose tissue and muscle (18); our studies demonstrated that similar increases in LPL mass and activity occur in the arterial wall. Thus, increased LPL resulting from SAT feeding may increase LDL uptake and/or retention by "bridging" to cell surface or ECM in the arterial wall, a condition that favors SU (9).

Babaev et al. (25) indicated that focal expression of LPL by macrophages in the arterial wall promotes atherosclerosis. Our studies indicate that the SAT diet modulates arterial LPL levels and distribution, especially in the medial layer. Of interest, increased LPL paralleled BODIPY- $\mathrm{C}_{12}$ fluorescence patterns in which more BODIPY-C 12 was seen in medial layers in C57BL/ 6 and apoE $\mathrm{E}^{-/-}$mice fed the SAT at 4 weeks and at 12 weeks, at least in C57BL/ 6 mice. Higher and more dispersed LPL expression along with deeper LDL$\mathrm{CE}$ infiltration into a medial layer may relate to increases in certain

cell populations (e.g., macrophages) in the arterial wall, but this remains to be investigated. More widely distributed LPL present within the arterial wall would likely anchor more LDL and increase SU. Increased arterial LPL could also potentially lead to focal increases in TG hydrolysis, which could further stimulate SU by elevating FFA concentrations focally near individual cells, a condition that, at least in vitro, enhances SU (13).

Changes in LPL relate to increased arterial SU in $\mathrm{C} 57 \mathrm{BL} / 6$ mice and apoE $\mathrm{E}^{-/-}$mice that have no atherosclerotic lesions. Other mechanisms may also increase arterial SU in apoE $\mathrm{E}^{-/}$mice with extensive atherosclerosis. After 12 weeks of feeding, there was a markedly higher contribution of SU in apoE $\mathrm{E}^{-/-}$than in $\mathrm{C} 57 \mathrm{BL} / 6$ mice, yet we were unable to demonstrate overexpression and wider distribution of arterial LPL in situ in these mice. The inability to visualize LPL in apoE $\mathrm{E}^{-/-}$mice is likely associated, in part, with significantly higher noncellular debris within the arterial wall that prevents effective $\mathrm{Ab}$ penetration and interaction with LPL.

We suggest that another contributing factor for $\mathrm{SU}$ in apoE $\mathrm{E}^{-/-}$ mice is the presence of atherosclerosis. Eight-week-old apoE $\mathrm{E}^{-/-}$mice fed a chow or SAT diet for 4 weeks had less arterial cholesterol delivery via SU compared with older apoE-/- mice on a SAT diet. Since 1 major difference between the younger versus older apoE $\mathrm{E}^{-/}$ mice was the extent and severity of atherosclerosis, it is likely that other processes involved in atherosclerosis also contribute to increased LDL-CE uptake via SU. One example could be changes in arterial wall proteoglycans, key modulators of SU in vitro (9). Moreover, FFAs have been shown to redistribute cholesterol pools in macrophages and promote proteoglycan synthesis; both can modulate SU $(9,13)$. Furthermore, certain FFAs modulate expression of PPAR and SREBP, important regulators of lipid metabolism, and alter cholesterol homeostasis, which also could affect SU (13). Since LPL is regulated by both PPAR $\alpha$ and PPAR $\gamma$, which are expressed in cells such as macrophages and smooth muscle cells in the arterial wall, studies on how changes in PPARs expression influence arterial LDL uptake and SU will be of interest.

Our studies indicate that SU can be an important contributor to arterial CE delivery and that this process is modified by diet. There are some limitations to our studies. For example, complete definition of the effects of different factors on SU, such as changes in cholesterol and FFA levels, extent of atherosclerosis, or duration of feeding periods, was not possible in our mouse models, since these factors change concomitantly and also can induce other structural and biochemical alterations in lipid metabolism and atherosclerosis development. Also, our SU measurements related to a cellular process that increased intracellular LDL-CE uptake but not apoB uptake. In apoE $\mathrm{E}^{-/-}$mice that are fed a chow or SAT diet for 12 weeks, significant amounts of LDL uptake and trapping can occur in ECM. Although our studies could not quantitatively determine how much intracellular versus extracellular trapping of LDL contributed to SU, we expect that deposition of whole LDL particles extracellularly would lead to increased accumulation of both LDL-CE and apoB; this would 
underestimate actual cellular SU, as it would decrease the ratios of LDL-CE to apoB.

In summary, multiple factors can interact to mediate SU and CE delivery to the arterial wall. These include blood cholesterol levels, diet, and atherosclerosis itself. Since LDL-CE delivery via SU increases proportionally to the extent of atherosclerosis, these interrelationships may represent "vicious cycles," particularly when other risk factors for cardiovascular disease are present, such as elevated plasma cholesterol levels. While further studies are needed, our data suggest that an atherogenic SAT diet may accentuate SU pathways to accelerate initial development of atherosclerosis. Increases in arterial wall LPL appear to be an important cofactor for this process. With progression of atherosclerosis, cholesterol delivery to the arterial wall will be increased by various nonclassical pathways including SU, leading to more complicated atherosclerotic lesions. Specific interventions to decrease the contribution of $\mathrm{SU}$ in cholesterol delivery to the arterial wall may be of value.

\section{Methods}

Sodium [ $\left.{ }^{125} \mathrm{I}\right]$ iodide was purchased from Dupont NEN. $\left[{ }^{3} \mathrm{H}\right] \mathrm{CEt}$ was purchased from Amersham Biociences. TC was kindly provided by Franz Rinninger (Universität Hamburg, Hamburg, Germany). BODIPY- $\mathrm{C}_{12}$ and ALEXA were purchased from Invitrogen Corp. Rabbit anti-mouse SR-BI Abs were purchased from Novus Biologicals Inc. Rabbit polyclonal antihuman LPL Abs were kindly provided by Ira Goldberg (Columbia University, New York, New York, USA). C57BL/6 mice were purchased from The Jackson Laboratory, and apoE $\mathrm{E}^{-/-}$mice (15) were maintained in our breeding colonies. All experimental procedures were approved by Columbia University's Institutional Animal Care and Use Committee.

LDL preparation and labeling. LDL $(\mathrm{d}=1.025-1.055 \mathrm{~g} / \mathrm{ml})$ was isolated from samples from normolipidemic humans by sequential ultracentrifugation. We separated LDL at the narrower density gradient range, so that the isolated LDL was void of apoE contamination. We labeled LDL with $\left[{ }^{3} \mathrm{H}\right] \mathrm{CEt}$ and nondegradable [125I]TC as previously described (9). Briefly, a glass tube was evenly coated with $200 \mu \mathrm{Ci}\left[{ }^{3} \mathrm{H}\right] \mathrm{CEt}$, and $2-3 \mathrm{mg}$ of isolated human LDL was incubated with human delipidated plasma $(\mathrm{d}<1.210 \mathrm{~g} / \mathrm{ml})$, a source of CE transfer protein, in the presence of neomycin sulfate for 48-72 hours to allow incorporation of $\left[{ }^{3} \mathrm{H}\right] \mathrm{CEt}$ into the LDL core. Labeled LDL was separated from plasma by ultracentrifugation and labeled with ${ }^{125}$ [TC] after iodination of TC with IODO-GEN (Pierce Biotechnology Inc.) and activation using cyanuric chloride $(9,26)$. The calculated cholesterol/protein ratios of LDL ranged from 1.6 to $1.8(n=20)$, with a typical specific activity of $10-50$ and $52-230 \mathrm{dpm} / \mathrm{ng}$ LDL protein for ${ }^{3} \mathrm{H}$ and ${ }^{125} \mathrm{I}$, respectively.

For fluorescent microscope studies, LDL was labeled with fluorescent BODIPY- $\mathrm{C}_{12}$, a CE analog, and ALEXA to trace LDL apoB (27). Briefly, 3 $\mathrm{mg} L \mathrm{LDL}$ was incubated with delipidated human plasma in a tube that was evenly coated with $1 \mathrm{mg}$ BODIPY- $\mathrm{C}_{12}$. After 24 hours incubation at $37^{\circ} \mathrm{C}$, LDL was re-isolated by sequential gradient ultracentrifugation, and apoB was then labeled with the ALEXA fluorophore according to the manufacturer's method. Isolated LDL was applied to a Sepharose CL-2B gel filtration column (Sigma-Aldrich) (28) to ensure that BODIPY-C $C_{12}$ and ALEXA fluorescence labeling did not alter LDL homogeneity and co-eluted with the LDL fraction (as determined by fluorometry). In separate experiments, fluorescent-labeled LDL cell binding and uptake and LDL-CE SU were compared with those of radiolabeled [ $\left.{ }^{125} \mathrm{I}\right] \mathrm{LDL}$ in vitro in cultured fibroblasts and showed similar results in both LDL binding and cell association (data not shown). Our labeling procedures did not modify LDL properties or induce LDL oxidation $(13,29)$.

Feeding protocols and blood lipid analyses. Four-week-old male C57BL/6 and apoE-/- mice weighing $15-18 \mathrm{~g}$ were fed a normal chow (4.5\% fat) or a SAT diet for 4 and 12 weeks ( $n>10$ for each group). The SAT diet consisted of $21 \%$ fat (wt/wt; $71 \%$ saturated fat from coconut oil, $19 \%$ monounsaturated fat from olive oil, and $9 \%$ polyunsaturated fats from safflower and corn oil; Harlan Teklad). The SAT diet contained $0.2 \%$ cholesterol (wt/wt). During the feeding periods, blood was obtained weekly for 4 weeks by retro-orbital blood sampling and then every 2 weeks for the remainder of the feeding period.

Blood FFA levels were determined using the Wako NEFA C kit (Wako Chemicals $\mathrm{GmbH}$ ), and TG and total cholesterol levels were determined using enzymatic kits (Roche Diagnostics Corp.) according to the manufacturers' procedures (28).

Blood clearance of injected LDL. At the end of the feeding period, a bolus of double-labeled human LDL (200 $\mu$ g protein) was injected through the saphenous vein, with the mice continuing on their respective diets for 24 hours ( $n=5$ for each group). Blood LDL clearance was assessed by independently measuring $\left[{ }^{3} \mathrm{H}\right] \mathrm{CEt}$ and $\left[{ }^{125} \mathrm{I}\right] \mathrm{apoB}$ in blood samples $(20 \mu \mathrm{l})$ at $30 \mathrm{sec}-$ onds after LDL injection. Comparison of radioactivity present in blood 30 seconds after LDL injection and initial injected radioactivity dose revealed that more than $98 \%$ of injected LDL was recovered from the circulation at 30 seconds. Thus, this value was used to calculate the percent recovery of injected LDL at the later time points (13). Additional blood samplings were performed at $1,2,4,8,12$, and 24 hours. More than $98 \%$ of injected LDL was cleared by 24 hours after injection (9). Radioactivity in the blood was expressed as the percent of injected dose remaining in whole blood. Whole blood volume for each mouse was calculated as $4.9 \%$ of body weight. FCRs were calculated on the basis of first-order linear kinetics during the first 8 hours after LDL injection.

LDL uptake in organs and tissues. Mice fed a chow or SAT diet were injected with $\left[{ }^{3} \mathrm{H}\right] \mathrm{CEt} /\left[{ }^{125} \mathrm{I}\right] \mathrm{TC}-\mathrm{labeled}$ human LDL (200 $\mu \mathrm{g}$ protein), and the mice were continued on their respective diets for 24-48 hours. Then the vascular system was thoroughly perfused with saline containing heparin $(3 \mathrm{IU} / \mathrm{ml})$, and tissues and aortas from these mice were isolated for determination of LDL-CE ([ $\left.\left.{ }^{3} \mathrm{H}\right] \mathrm{CEt}\right)$ and whole-particle ([125 I TC) uptake. The aorta from the aortic arch to abdominal aorta was assayed for radioactivity. We consistently observed reduced hepatic uptake of injected LDL in apoE-/- mice, which was presumably due to dilution of injected LDL and/or competition with increased endogenous lipoproteins (see Results and Table 1). Thus, LDL-CE delivery via SU was expressed as the mean of ${ }^{3} \mathrm{H} /{ }^{125}$ I ratios (>1 indicates $\mathrm{SU}$ ) or percent LDL-CE delivery via SU relative to LDL-CE uptake to eliminate the potential confounders of labeled LDL dilution and/or competition. LDL-CE delivery was determined directly from $\left[{ }^{3} \mathrm{H}\right] \mathrm{CEt}$ uptake and was compared with estimated LDL-CE delivery calculated based on [ $\left.{ }^{125} \mathrm{I}\right] \mathrm{TC}-\mathrm{apoB}$ uptake using specific activity of ${ }^{125} \mathrm{I}$ per $\mu \mathrm{g}$ LDL-CE (9). The contribution of SU to total LDL-CE uptake was determined by subtracting the estimated LDL-CE delivery from total LDL-CE uptake and was then expressed as the percent LDL-CE delivered via SU relative to the total LDL-CE delivery.

Since radioactivity in the aorta was substantially lower than that in the blood compartment, specific control experiments were performed to assess the possibility of potential overestimation of arterial ${ }^{3} \mathrm{H}$ and ${ }^{125} \mathrm{I}$ uptake associated with blood contamination. Isolated arteries were extensively washed with saline containing heparin and EDTA to remove any surfacebound blood contaminants. After washing, there was no reduction in arterial radioactivity, which indicated that ${ }^{3} \mathrm{H}$ and ${ }^{125} \mathrm{I}$ traced LDL-CE and apoB internalized by cells within the arterial wall and not LDL adhering to the surface of the arterial wall of $\mathrm{C} 57 \mathrm{BL} / 6$ and $\mathrm{apoE}^{-/-}$mice with or without atherosclerosis. In addition, aortas were isolated at 48 hours rather than for 24 hours (as in most experiments) from 5 mice to determine whether accumulated radioactivity in the arterial wall was released back into blood compartment. Arterial radioactivity at 48 hours was unchanged compared 
with that at 24 hours, which indicated that radioactivity in the arterial wall did not efflux from the arteries after LDL was internalized in arteries. Thus, use of nonhydrolyzable tracers for both apoB and LDL-CE eliminated or reduced the potential confounders associated with extracellular aggregation and/or different turnover rates for apoB and LDL-CE (e.g., following degradation in the arterial wall), as these would have affected LDL-CE/apoB ratios for SU determination.

Determination of fluorescent $L D L$ uptake in the arterial wall. At the end of the feeding periods as described above, mice were injected with $200 \mu \mathrm{g}$ double fluorescent-labeled LDL (BODIPY- $\mathrm{C}_{12}$ and ALEXA-apoB). Eight hours after injection, mice were extensively perfusion fixed with $6 \%$ paraformaldehyde solution. Isolated aortas were sectioned $(14 \mu \mathrm{m})$ with a cryomicrotome after being embedded in Tissue-Tek OCT (Sakura Finetek U.S.A. Inc.). BODIPY- $\mathrm{C}_{12}$ and ALEXA fluorescence were observed with a laser scanning confocal microscope (Zeiss LSM-510) equipped with an image-capture device at $\times 20$ and $\times 100$ magnification. The microscope settings were 488 and $543 \mathrm{~nm}$ (excitation wavelength) and 475-575 and $560 \mathrm{~nm}$ (emission wavelength) for BODIPY- $\mathrm{C}_{12}$ and ALEXA, respectively. All samples were captured with the same exposure times for consistency. To determine BODIPY- $C_{12} /$ ALEXA ratios, we quantitated fluorescence intensity in the intima-medial layers of the aortic arch (proximal) and abdominal aortas (distal) using ImageJ version 1.31 (NIH; http://rsb.info.nih.gov/ij/). These quanitifications were performed on at least 5 different sections of both proximal and distal aortas in each mouse ( $n=5$ for each mouse group) and the mean of ratios determined for each group.

Oil red O staining. Sectioned aortas $(n>10)$ were immersed in $60 \%$ isopropanol for 30 second and then in oil red O solution for 1 minute to stain neutral lipids (TGs and CEs). Stained samples were washed extensively before being observed under a Nikon Labophot 2 microscope (30). Whole $(\times 10-20)$ and partial artery images $(\times 200-400)$ were captured at different magnifications. Image analysis software (Image Pro Plus 3.0; EPIX Inc.) was used to separate stained from nonstained regions within the intimamedial layer of particular artery samples and to measure their areas in square micrometers $\left(\mu \mathrm{m}^{2}\right)$, which were compared with the total areas of the arterial wall, a procedure that has been used as standard quantification method for atherosclerosis (31).

Immunohistochemistry. At the end of the feeding period, mice were sacrificed, and the aorta was immediately removed. Isolated arteries were fixed with $6 \%$ parafomaldehyde and cross-sectioned with a cryomicrotome. Sectioned arteries were washed and incubated with polyclonal antimouse LPL Ab (1:100), and then incubated with a secondary anti-rabbit IgG (1:1000) conjugated with alkaline phosphatase. The secondary Ab was stained using an Alkaline Phosphatase Staining Kit (Sigma-Aldrich) according to the manufacturer's instructions to determine arterial LPL levels and localization. Images of stained arteries were captured at $\times 400$ magnification with a Nikon Labophot 2 microscope equipped with a Sony CCD-Iris RGB color video camera (30). Captured images of the light microscope were measured for LPL staining intensity in the intimalmedial layer of the arterial wall as described above. Results from at least 10 different sections of the same proximal aorta per mouse were averaged in each group $(n=4-6)$ after normalization to the quantitated area size of the intima-medial layer.

Western blots and LPL activity in the arterial wall. Isolated aortas from different mice fed a chow or SAT diet $(n=3-5)$ were homogenized with a Dounce homogenizer in the presence of saline containing protease inhibitors (Roche Diagnostics Corp.), which was followed by a brief sonication. Thirty micrograms artery protein was separated with $8 \%$ nongradient SDSPAGE and transferred to Duralon membranes for immunoblot analyses for LPL and SR-BI expression. Titers of primary Abs used were 1:500 and 1:1,000, respectively. HRP-conjugated anti-mouse or anti-rabbit secondary Abs $(1: 50,000)$ were detected using SuperSignal (Pierce Biotechnology Inc.) (14) and exposed to X-ray films according to the manufacturer's instructions. Bands were measured by densitometry and normalized for $\beta$-actin levels in corresponding samples. Results are expressed as the relative density changes in SAT-fed mice compared with control chow-fed mice in each experimental group. From the same arterial homogenates, an aliquot was used to measure LPL activity using radiolabeled $\left[{ }^{3} \mathrm{H}\right]$ Intralipid (Intralipid from Fresenius Kabi Clayton) as described previously (32).

Statistical analyses. One-way ANOVA, 2-tailed Student's $t$ tests, and linear regression were used to determine statistical significance at the level of $P<0.05$.

\section{Acknowledgments}

This work was supported by NIH grants DK60497 (to T. Seo) and HL40404 (to R.J. Deckelbaum) and American Heart Association Grant-in-Aid 0255656N (to T.S. Worgall).

Received for publication December 27, 2004, and accepted in revised form May 24, 2005.

Address correspondence to: Toru Seo, Institute of Human Nutrition, Columbia University, 630 West 168th Street, PH1512, New York, New York 10032, USA. Phone: (212) 305-3961; Fax: (212) 305-3079; E-mail: ts227@columbia.edu.
1. Brown, M.S., and Goldstein, J.L. 1986. A receptor mediated pathway for cholesterol homeostasis. Science. 232:34-47.

2. Calvo, D., Gomez-Coronado, D., Suarez, Y., Lasuncion, M.A., and Vega, M.A. 1998. Human CD36 is a high affinity receptor for the native lipoproteins HDL, LDL, and VLDL. J. Lipid Res. 39:777-788.

3. Rigotti, A., Acton, S.L., and Krieger, M. 1995. The class B scavenger receptors SR-B1 and CD36 are receptors for anionic phospholipids. J. Biol. Chem. 270:16221-16224.

4. Han, J., Hajjar, D.P., Febbraio, M., and Nicholson, A.C. 1997. Native and modified low density lipoproteins increase the functional expression of the macrophage class B scavenger receptor, CD36. J. Biol. Chem. 272:21654-21659.

5. Febbraio, M., et al. 2000. Targeted disruption of the class B scavenger receptor CD36 protects against atherosclerotic lesion development in mice. J. Clin. Invest. 105:1049-1056.

6. Seo, T., and St. Clair, R.W. 1997. Heparan sulfate proteoglycans mediate internalization and deg- radation of beta-VLDL and promote cholesterol accumulation by pigeon macrophages. J. Lipid Res. 38:765-779.

7. Green, S.R., and Pittman, R.C. 1991. Selective uptake of cholesteryl esters from low density lipoproteins in vitro and in vivo. J. Lipid Res. 32:667-678.

8. Rinninger, F., Brundert, M., Jäckle, S., Kaiser, T., and Greten, H. 1995. Selective uptake of low-density lipoprotein-associated cholesteryl esters by human fibroblasts, human HepG2 hepatoma cells and J774 macrophages in culture. Biochim. Biophys. Acta. 1255:141-153.

9. Seo, T., et al. 2000. Lipoprotein lipase-mediated selective uptake from low density lipoprotein requires cell surface proteoglycans and is independent of scavenger receptor class B type 1. J. Biol. Chem. 275:30355-30362.

10. Krieger, M. 1999. Charting the fate of the "good cholesterol": identification and characterization of the high-density lipoprotein receptor SR-BI [review]. Annu. Rev. Biochem. 68:523-558.

11. Temel, R.E., et al. 1997. Scavenger receptor class
B, type I (SR-BI) is the major route for the delivery of high density lipoprotein cholesterol to the steroidogenic pathway in cultured mouse adrenocortical cells. Proc. Natl. Acad. Sci. U. S. A. 94:13600-13605.

12. Ji, Y., et al. 1997. Scavenger receptor BI promotes high density lipoprotein-mediated cellular cholesterol efflux. J. Biol. Chem. 272:20982-20985.

13. Seo, T., et al. 2002. Selective uptake from LDL is stimulated by unsaturated fatty acids and modulated by cholesterol content in the plasma membrane: role of plasma membrane composition in regulating non-SR-BI-mediated selective lipid transfer. Biochemistry. 41:7885-7894.

14. Worgall, T.S., Sturley, S.L., Seo, T., Osborne, T.F., and Deckelbaum, R.J. 1998. Polyunsaturated fatty acids decrease expression of promoters with sterol regulatory elements by decreasing levels of mature sterol regulatory element-binding protein. J. Biol. Chem. 273:25537-25540.

15. Osada, J., Joven, J., and Maeda, N. 2000. The value of apolipoprotein E knockout mice for studying 
the effects of dietary fat and cholesterol on atherogenesis. Curr. Opin. Lipidol. 11:25-29.

16. Rinninger, F., et al. 1998. Lipoprotein lipase mediates an increase in the selective uptake of high density lipoprotein-associated cholesteryl esters by hepatic cells in culture. J. Lipid Res. 39:1335-1348.

17. Haug, A., and Hostmark, A.T. 1987. Lipoprotein lipases, lipoproteins and tissue lipids in rats fed fish oil or coconut oil. J. Nutr. 117:1011-1017.

18. Corey, J.E., and Zilversmit, D.B. 1977. Effect of cholesterol feeding on arterial lipolytic activity in the rabbit. Atherosclerosis. 27:201-212.

19. Webb, N.R., et al. 2002. Overexpression of SR-BI by adenoviral vector promotes clearance of apoA-I, but not apoB, in human apoB transgenic mice. J. Lipid Res. 43:1421-1428.

20. Webb, N.R., de Beer, M.C., de Beer, F.C., and van der Westhuyzen, D.R. 2004. ApoB-containing lipoproteins in apoE-deficient mice are not metabolized by the class B scavenger receptor BI. J. Lipid. Res 45:272-280.

21. Swarnakar, S., Reyland, M.E., Deng, J., Azhar, S., and Williams, D.L. 1998. Selective uptake of low density lipoprotein-cholesteryl ester is enhanced by inducible apolipoprotein E expression in cultured mouse adrenocortical cells. J. Biol. Chem. 273:12140-12147.

22. Portman, O.W., and Alexander, M. 1985. Metabolism of [125I]tyramine cellobiose-labeled low density lipoproteins in squirrel monkeys. Atherosclerosis. 56:283-299.

23. Tabas, I. 2004. Apoptosis and plaque destabilization in atherosclerosis: the role of macrophage apoptosis induced by cholesterol. Cell Death Differ. 11(Suppl. 1):S12-S16.

24. Rutledge, J.C., Woo, M.M., Rezai, A.A., Curtiss, L.K., and Goldberg, I.J. 1997. Lipoprotein lipase increases lipoprotein binding to the artery wall and increases endothelial layer permeability by formation of lipolysis products. Circ. Res. 80:819-828.

25. Babaev, V.R., et al. 1999. Macrophage lipoprotein lipase promotes foam cell formation and atherosclerosis in vivo. J. Clin. Invest. 103:1697-1705.

26. Schwenke, D.C., and Carew, T.E. 1989. Initiation of atherosclerotic lesions in cholesterol-fed rabbits. I. Focal increases in arterial LDL concentration precede development of fatty streak lesions. Arteriosclerosis. 9:895-907.
27. Reaven, E., Tsai, L., and Azhar, S. 1996. Intracellular events in the "selective" transport of lipoprotein-derived cholesteryl esters. J. Biol. Chem. 271:16208-16217.

28. Qi, K., et al. 2002. Omega-3 triglycerides modify blood clearance and tissue targeting pathways of lipid emulsions. Biochemistry. 41:3119-3127.

29. Galeano, N.F., Al-Haideri, M., Keyserman, F., Rumsey, S.C., and Deckelbaum, R.J. 1998. Small dense low density lipoprotein has increased affinity for LDL receptor-independent cell surface binding sites: a potential mechanism for increased atherogenicity. J. Lipid Res. 39:1263-1273.

30. Feng, B., et al. 2003. Niemann-Pick C heterozygosity confers resistance to lesional necrosis and macrophage apoptosis in murine atherosclerosis. Proc. Natl. Acad. Sci. U. S. A. 100:10423-10428.

31. Nunnari, J.J., Zand, T., Joris, I., and Majno, G. 1989. Quantitation of oil red O staining of the aorta in hypercholesterolemic rats. Exp. Mol. Pathol. 51:1-8.

32. Yagyu, H., et al. 2003. Lipoprotein lipase (LpL) on the surface of cardiomyocytes increases lipid uptake and produces a cardiomyopathy. J. Clin. Invest. 111:419-426. doi:10.1172/JCI200316751. 